\title{
Algorithm for bearings-only Passive Localization Based on UKF
}

\author{
Geng Xiaoming*, Suo Yujun, Yang Hongyue
}

Tianjin Jinhang Computing Technology Research Institute

653618810@qq.com

${ }^{*}$ Corresponding author

Keywords: passive localization, bearings-only, unscented Kalman filter

\begin{abstract}
In order to improve the precison and the speed of passive localization ,as well as meet the high applicable values requirements, a passive localization algorithm based on the Unscented Kalman Filtering is proposed. The main idea is to use the Filtering algorithm to solve the sensor information fusion problems; and utlize the Unscented Kalman Filtering algorithm to eliminate the gauss noise of the nonlinear state-space equations, thus achieving fast and high-precision localization .Simulation results show that the algorithm has good feasibility, high precision, high speed and high applicable values.
\end{abstract}

\section{Introduction}

The airborne passive location and tracking system, which intercepts the electromagnetic waves emitted by targets, has played a major role in avionic reconnaissance and anti-radiation unmanned aerial vehicle (UAV) homing guidance. Passive location technology is prior to active location technology in far distance, secret receiving and low probability of detection. The passive location technology has broad prospects in measurement and control of spacecraft, satellite location, etc. Single observer passive location technology works with only one observing platform. [1 2] Its superiorities are less equipment and lower cost. But this technology is difficult to realize because the information obtained is insufficient compared to multi-observer location technology. Traditional methods of the passive location technology include bearing only (BO), time only (TO), Doppler only (DO), bearing/ time (BT) and bearing/Doppler (BD). The shortcomings of these methods are long locating time, low precision, high sensitivity to direction measurement errors [3].

The accuracy of the emitter's location is determined by the measurement precision and the geometrical constraints of the observer relative to the emitter [4]. The conventional widely-used passive location technique with bearings-only measurements. With an angular accuracy of nearly $1^{\circ}-2^{\circ}$ at present, the avionic device is found to be hard to enhance the angular accuracy further due to the limitation of the antenna aperture.

\section{The principle of bearings-only passive location}

Passive location is a technique that locates the target emitter through receiving its radio wave without emitting any radio signals during operation. It is characterized by long effective distance, electromagnetic silence,and undetectable possibility. It has long been an indispensable part of an integrated air defense system and long distance airborne warning system either on land or on sea. Meanwhile, it is a key means to develop marine, aeronautic, astronautic, detection, tracking, and geographical sciences and technologies.

It is required to acquire parameters carrying the location information of the target emitter from the received signals by means of the kinematics-based passive locating and tracking algorithm.All the methods focused the attention on the full utilization of the information involved in radio signals about the position of the target emitter so as to quickly and accurately locate the emitter itself. This makes accurate acquisition of parameters one of the key techniques in passive locating system. 


\subsection{Bearings-only passive location model}

The angle relationship between the aircraft and the object is plotted in Figure 1. the position of the object is $X_{m}^{(k)}$,the coordinate position of aircraft is $X_{f}^{(k)}$, the azimuth angle between aircraft and object is $\theta(k)$.

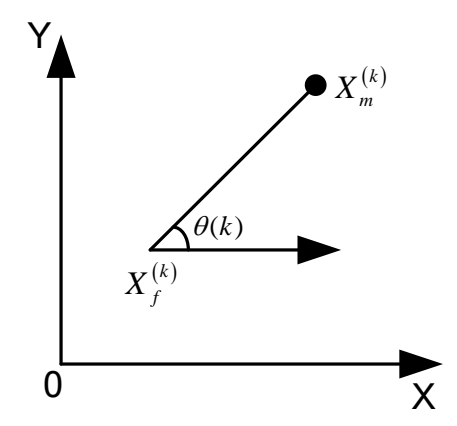

Figure 1 plot of bearings-only passive location

The positions of object and aircraft at time $\boldsymbol{k}$ :

$$
\begin{aligned}
& X_{m}^{(k)}=\left[\begin{array}{ll}
X_{m}^{(k)} & y_{m}^{(k)}
\end{array}\right] \\
& X_{f}^{(k)}=\left[\begin{array}{ll}
X_{f}^{(k)} & y_{f}^{(k)}
\end{array}\right]
\end{aligned}
$$

the azimuth angle between aircraft and object $\theta(k)$ at time $\boldsymbol{k}$ :

$$
\theta(k)=\tan ^{-1}\left(\frac{y_{f}^{(k)}-y_{m}^{(k)}}{x_{f}^{(k)}-x_{m}^{(k)}}\right)+v(k)
$$

Where, $v(k)$ is measurement noise.

\subsection{The bearings-only passive location algorithm based on UKF}

The Kalman filter has been widely applied in multi-sensor integration application for decades and become a standard approach to estimate the system state.The EKF can be difficult to tune and often gives unreliable estimates if the system nonlinearities are severe. In the EKF, we use only the first term of this expansion to approximate the covariance of the estimation error. The Unscented Kalman Filter (UKF), introduced by Julier and Uhlman, is another probabilistic approach to approximate the state distribution by Gaussian random variable [5]. It utilizes the Unscented Transform (UT) to estimate the system state which undergoes a nonlinear transformation [6], [7]. Unlike the PF, the UKF requires only a minimal set of samples, namely the sigma points, to propagate through the nonlinear equation. The predicted mean and covariance are calculated accurately by the transformed sigma points at least to the second order of Taylor expansion [8], [9]. Different sampling strategies of sigma points have been proposed to improve the estimation efficiency [8], [10]. The bound radius of the sigma points, however, affects the estimation accuracy. The Scaled Unscented Transformation (SUT) is an approach to reduce the spread of the sigma points and decrease the nonlocal effect in state estimation .

The UKF can be summarized as follows.

1) Parameters

$$
\begin{aligned}
\gamma & =\sqrt{n+\lambda} \\
\omega_{0}^{(m)} & =\frac{\lambda}{n+\lambda}
\end{aligned}
$$




$$
\begin{gathered}
\omega_{0}^{(c)}=\omega_{0}^{(m)}+\left(1-\alpha^{2}+\beta\right) \\
\omega_{i}^{(c)}=\omega_{i}^{(m)}=\frac{1}{2(n+\lambda)}, i=1, \ldots, 2 n \\
\lambda=\alpha^{2}(n+k)-n
\end{gathered}
$$

where, $\lambda$ is a scale factor; $\alpha$ is the scale factor which determines the bound radius of the sigma points; if the state is Gaussian, then $\beta=2 ; k$ affects the third and higher order terms of the Taylor expansion, generally, $k=0$.

2) Sigma points $\xi_{\mathrm{k}-1}^{(i)}(i=1,2, \ldots, 4)$

$$
\left\{\begin{array}{l}
\xi_{\mathrm{k}-1}^{(0)}=\widehat{\mathrm{X}}_{\mathrm{k}-1} \\
\xi_{\mathrm{k}-1}^{(\mathrm{i})}=\hat{\mathrm{X}}_{\mathrm{k}-1}+\left(\sqrt{(n+\lambda) \mathrm{P}_{\mathrm{k}-1}}\right)_{\mathrm{i}}, \mathrm{i}=1,2 \\
\xi_{\mathrm{k}-1}^{(\mathrm{i})}=\widehat{\mathrm{X}}_{\mathrm{k}-1}-\left(\sqrt{(n+\lambda) \mathrm{P}_{\mathrm{k}-1}}\right)_{\mathrm{i}-2}, \mathrm{i}=3,4
\end{array}\right.
$$

3) The time update equations are given as follows:

The Sigma points $\xi_{\mathrm{k}}^{(i)}(i=1,2,3,4)$ are propagated as follows:

$$
\begin{gathered}
\xi_{\mathrm{k}}^{(\mathrm{i})}=\xi_{\mathrm{k}-1}^{(\mathrm{i})}, i=0,1, \ldots, 4 \\
\hat{X}_{\mathrm{k}, \mathrm{k}-1}=\sum_{i=0}^{4} \omega_{i}^{(m)} \xi_{\mathrm{k}}^{(i)} \\
P_{\mathrm{k}, \mathrm{k}-1}=\sum_{i=0}^{4} \omega_{i}^{(c)}\left(\xi_{\mathrm{k}}^{(i)}-\hat{X}_{\mathrm{k}, \mathrm{k}-1}\right)\left(\xi_{k}^{(i)}-\widehat{X}_{\mathrm{k}, \mathrm{k}-1}\right)^{T}
\end{gathered}
$$

4) The measurement update equations are given as follows:

$$
\begin{gathered}
\chi_{i, k, k-1)}=h\left(\xi_{\mathrm{k}}^{(\mathrm{i})}\right) \\
\hat{\theta}_{k, k-1}=\sum_{i=0}^{4} \omega_{i}^{(m)} \chi_{i,(k, k-1)} \\
P_{\tilde{\theta}_{\mathrm{k}}}=\sum_{i=0}^{4} \omega_{i}^{(c)}\left(\chi_{i,(k, k-1)}-\hat{\theta}_{k, k-1}\right)\left(\chi_{i,(k, k-1)}-\hat{\theta}_{k, k-1}\right)^{T}+V \\
P_{\mathrm{X}_{\mathrm{k}} \theta_{k}}=\sum_{i=0}^{4} \omega_{i}^{(c)}\left(\xi_{i,(k, k-1)}-\hat{X}_{\mathrm{k}, \mathrm{k}-1}^{a}\right)\left(\chi_{i,(k, k-1)}-\hat{\theta}_{k, k-1}\right)^{T}
\end{gathered}
$$

5) The filter update as follows:

$$
\begin{gathered}
\mathrm{K}_{k}=P_{\mathrm{X}_{\mathrm{k}} \theta_{k}} P_{\tilde{\theta}_{\mathrm{k}}}^{-1} \\
\widehat{X}_{\mathrm{k}}=\widehat{X}_{\mathrm{k}, \mathrm{k}-1}+K_{k}\left(\theta_{k}-\widehat{\theta}_{k, k-1}\right) \\
P_{k}=P_{k, k-1}-K_{k} P_{\tilde{\theta}_{\mathrm{k}}} K_{k}^{T}
\end{gathered}
$$




\section{Simulation}

\subsection{Parameters and Models}

The simulation assumes that the coordinate of the target is [120000,0], the original coordinate of the UAV is $[100,100]$,the angle is $45^{\circ}$,the velocity is $[200,200] \mathrm{m} / \mathrm{s}$, the measurement noise are white, zero-mean Gaussian stochastic processes with covariances $3^{\circ}$.

\subsection{The filter parameters}

The original state is :

$$
\widehat{X}_{g}^{(0)}=[100000,20000]
$$

The original error is :

$$
P_{g}^{(0)}=\left[\begin{array}{cc}
30000^{2} & 0 \\
0 & 30000^{2}
\end{array}\right]
$$

The other parameters are: $n=2, \alpha=0.1, \beta=2, k=0$.

\subsection{The simulation result}

Figure 2 shows the simulation result of the bearings-only passive location based on UKF.

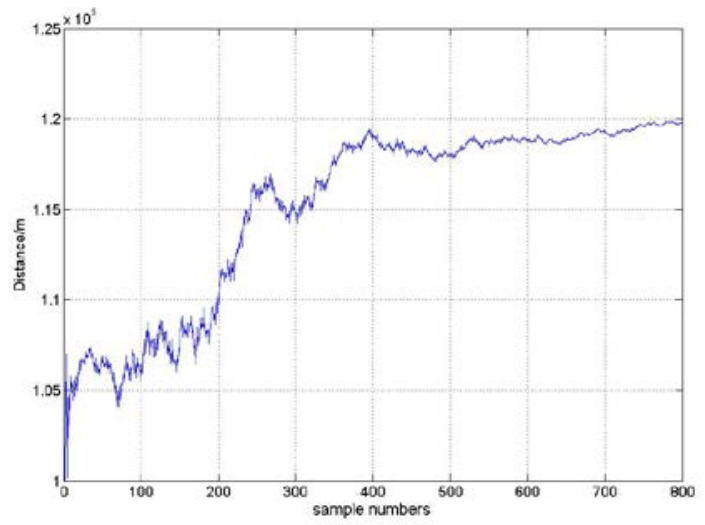

Figure 2 The simulation result of the passive location based on UKF

\section{Conclusions}

The algorithm for bearings-only passive location of the single observer based on UKF is analyzed. It is presented that the unscented Kalman filter (UKF)that reduces the linearization errors of the EKF. The Extended Kalman Filter, however, is straight forward and less sensitive to numerical stability relative to Unscented Kalman Filter. unscented transformations are more accurate than linearization for propagating means and covariances. Therefore, we simply replace the EKF equations with unscented transformations to obtain the UKF algorithm.Finally, we conclude that, using the UKF can provide significant improvement over the EKF.

\section{References}

[1] Rose M. C. Method for determing the optimum observer heading change in bearing-only passive emitter tracking[P]. United States Patent: 6801152, 2004.

[2] Guan X, Yi X, He Y. Research on unobservability problem for two-dimensional bearings-only target motion analysis[C]/Proceedings of International Conference on Intelligent Sensing and 
Information Processing, 2005: 56-60.

[3] Blair W D, Rice T R.A synchronous data fusion for target tracking with a multitasking radar and option sensor. SPIE. 1991, 1482: 234-245.

[4] Gan Q, H arrisC J. Com parison of two m easurement fus ion methods for Kalm and filter based multisensor data fusion. IEEE Transactions on Aerospace and Electronic Systems, 2001, 37(1):273 280.

[5] Simo Särkkä. On Unscented Kalman Filtering for State Estimation of Continuous-Time Nonlinear Systems[J]. IEEE Transactions on Automatic Control ,2007, 52(9):1631-1641.

[6] Stephen C, Felter N, Eva Wu. A relative navigation system for formation flight. IEEE Trans on Aerospace and Electronic Systems. 1997 ,33 (3) :958-967

[7] Hepner, S. A. R., and Geering, H. P., "Observability Analysis for Target Maneuver Estimation via Bearing-Only and Bearing-Rate-Only Measurements", J. of Guidance, Control, and Dynamics, Vol.13, No. 6, Nov.-Dec. 1990, pp. 977-983.

[8] Chang, W. T., and Lin, S. A., "Incremental Maneuver Estimation Model for Target Tracking", IEEE Trans.on Aerospace and Electronic Systems, Vol. 28, No.2, April 1992, pp. 439-451.

[9] Oshman, Y., and Shinar, J. "Using a Multiple Model Adaptive Estimator in a Random Evasion Missile/Aircraft Estimation”, in Proc. of the AIAA Guidance, Navigation and Control Conference, Portland, OR, Aug. 1999, AIAA Paper 99-4141.

[10] Stallard, D. V., “Angle-Only Tracking Filter in Modified Spherical Coordinates”, J. of Guidance, Control and Dynamics, Vol. 14, No. 3, May-June 1991, pp. 694-69. 